\title{
Deterministic Traffic Regulation with Decoder Buffer Constraints for Streaming Videos
}

\author{
Chu-Chuan Lee and Pao-Chi Chang \\ Department of Electrical Engineering, National Central University \\ Jongli City, Taiwan, R.O.C. \\ E-mail: \{cclee,pcchang \}@vaplab.ee.ncu.edu.tw
}

\begin{abstract}
Due to the burstiness property of compressed videos, an effective video traffic description scheme is important for efficient network bandwidth managements. This paper proposes an Elastically Deterministic Video Traffic Regulating mechanism (ED-VTR) that models and regulates the video traffic based on constraints of available decoder buffer space. In contrast to traditional methods, ED-VTR generates a transmission schedule that contains a series of monotonically decreasing bandwidth requests. Simulation results show that, using lower initial bandwidth and smaller decoder buffer requirement, ED-VTR can effectively reduce the complexity of bandwidth management and still hold advantages of traditional traffic smoothing and deterministic traffic modeling methods.
\end{abstract}

\section{Introduction}

With the success of Internet and the enhancement of network infrastructures, rich video applications are generated substantially. However, compressed videos often exhibit the burstiness property on a variety of time scales, and this will increase the complexity of resource management. In such a situation, unacceptable delay or loss may occur if the network directly reserves the bandwidth according to the average rate of video source. In contrast, the resource may always be underutilized if only the peak rate of video source is used in the resource management. Furthermore, from the network perspective, any halfway request for increasing reserved bandwidth may complicate resource management and cause unexpected packet loss or latency. Therefore, the efficiency of resource management significantly depends on the performance of traffic modeling schemes.

To date, many literatures have proposed various video traffic modeling methods to describe characteristics of video sources, such as the self-similar model [1] and empirical envelope model with linear concave approximation [2]. Basically, these schemes can be classified into two classes, the stochastic traffic modeling class and the deterministic traffic modeling class. Deterministic modeling methods such as [2] can provide loss free and bounded delay guarantees to the modeled traffic. In addition, deterministic model can be parameterized easily such that the implementation is more feasible than the stochastic approach. Therefore, this paper aims at related researches of deterministic traffic modeling.

Among those approaches, Pao and Sun [3] proposed a sliding-window rate-control scheme where the constraints of decoder buffer size and preloading time requirement were formulated. Wrege et al. [2] proposed an Empirical Envelope Modeling scheme, which is abbreviated as EEM in this paper, to characterize the video traffic. This scheme first used the worst-case description to get an empirical-envelope function $E^{*}$ for the actual cumulative transmitted data, and then generated a transmission schedule from $E^{*}$ in terms of the piece-wise linear concave upper approximation.

In this paper, an Elastically Deterministic Video Traffic Regulating mechanism (ED-VTR) which consists of two major parts is proposed. The target of the first part is to generate a series of monotonically decreasing bandwidth requirements, and the target of the second part is to execute an active frame skip algorithm at sender, which can avoid arbitrary skip operations while the decoder buffer size is insufficient. The above operations are named as traffic regulation because they not only execute the improved traffic modeling and smoothing works, but also execute the active frame skipping operations. Besides, it is noted that a QoS aware network in terms of the assistance of deterministic traffic modeling can provide the bounded delay and loss free guarantees to packets [2]. The rest of this paper is structured as follows. In section II, the proposed ED-VTR is described in detail. In section III, the active frame skip algorithm used in the ED-VTR is presented. Section IV shows and discusses simulation results. Finally, section $\mathrm{V}$ concludes this paper.

\section{Client-bounded deterministic traffic regulation}

This study considers a video streaming sequence with $N$ frames. The encoded bits of frame $i$ is denoted as $f_{i}$, for $i=1,2, \ldots, N$. Additionally, a discrete time model with a unit of frame-time is used in this paper. 
Based on characteristics of video decoding, ED-VTR first constructs an estimated function of cumulative consumed data, $L(\mathrm{n})$, which can be expressed as

$$
L(n)= \begin{cases}0, & 0 \leq n \leq D \\ \sum_{i=1}^{n-D} f_{i}, & D+1 \leq n \leq D+N\end{cases}
$$

where $D$ denotes the preloading time and $n=0$ represents the time instant that the first video packet arrives to decoder. From the client viewpoint, any possible transmission schedule for the client must always be larger than or equal to $L(\mathrm{n})$ to avoid buffer underflow. That is, $L(\mathrm{n})$ is the lower bound of the targeted transmission schedule. After constructing $L(\mathrm{n})$, the proposed scheme temporarily ignores the worst-case description that is used to obtain the empirical-envelope $E^{*}$ in [2], and directly utilizes the piece-wise linear concave upper approximation to bound $L(\mathrm{n})$. Meanwhile, a raw transmission schedule $S^{R}(n)$ comprising traffic parameter pairs $\left\{\left(\sigma_{i}^{R}, \rho_{i}^{R}\right) \mid \mathrm{i}=1,2, \ldots, M^{R}\right\}$ is generated, where $M^{R}$ represents the maximum number of parameter pairs, $\rho_{i}^{R}$ is the $i$-th token rate, and $\sigma_{i}^{R}$ is the $i$-th corresponding token depth.

However, as mentioned earlier, the decoder buffer space of terminals available for video applications may be insufficient, such as cellular handsets. To prevent possible decoder buffer overflow, this work further includes an upper bound into the generating process of transmission schedules. The upper bound $U(\mathrm{n})$ is then defined as

$$
U(n)=L(n)+B
$$

where $B$ represents the available decoder buffer space of clients. Meanwhile, a mature transmission schedule $S^{T}(n)$ for a targeted streaming video sequence is generated if the following two conditions hold:

1) $S^{T}(n)$ must always stay within $L(\mathrm{n})$ and $\mathrm{U}(\mathrm{n})$, i.e.

$$
L(n) \leq S^{T}(n) \leq U(n)
$$

2) The requested bandwidths generated by $S^{T}(n)$ must always be monotonically decreasing, that is

$$
\rho_{i}^{T}>\rho_{i+1}^{T}, \text { for } i=1,2, \cdots, M^{r}-1
$$

with

$$
\rho_{i}^{T}=\max _{a_{i}<\beta \leq b_{i}}\left\{\frac{L(\beta)-L\left(a_{i}\right)}{\beta-a_{i}}\right\}
$$

where $a_{\mathrm{i}}$ and $b_{\mathrm{i}}$ denote the beginning and end time of the $i$-th linear concave upper approximation, respectively. Moreover, $M^{T}$ represents the maximum number of mature parameter pairs generated by ED-VTR. The $\rho_{i}^{T}$ denotes the minimum transmission rate which does not cause the buffer underflow and overflow in interval $\left[a_{\mathrm{i}}, b_{\mathrm{i}}\right]$.

From (3) and (4), $S^{T}(n)$ generates a series of bandwidth requirements that exhibit the monotonically decreasing property; that is, no midway request for increasing bandwidth happens. This property can significantly simplify the resource management of network. After generating $S^{T}(n)$, this work now executes the worst-case description and obtains the empirical-envelope $E^{*}$ for $S^{T}(n)$. Interestingly, the obtained $E^{*}$ is identical to $S^{T}(n)$ itself. It is easy to conclude that $S^{T}(n)$ also can obtain the bounded delay and loss free guarantees if the network traces $S^{T}(n)$ to reserve bandwidth, since our proposed mechanism also belongs to the class of deterministic service models [2]. The detail algorithm of ED-VTR to generate a mature transmission schedule is shown in Fig. 1.

$$
\begin{aligned}
& \text { PROCEDURE Transmission_schedule ( ) \{ } \\
& i=1, \quad J_{1}=0 ; \\
& \text { WHILE }\left(N-J_{i}>0\right)\{ \\
& \rho_{i}^{R}=\max _{J_{i}<\beta \leqslant N}\left\{\frac{L(\beta)-L\left(J_{i}\right)}{\beta-J_{i}}\right\} ; \\
& \sigma_{i}^{R}=L\left(J_{i}\right)-\rho_{i}^{R} \times J_{i} ; \\
& P_{i}=\max \left\{\beta \mid \rho_{i}^{R} \cdot \beta+\sigma_{i}^{R}=L(\beta)\right\} ; \\
& \text { OUTPUT }\left(\rho_{i}^{R}, \sigma_{i}^{R}, J_{i}, P_{i}\right) \text {; } \\
& J_{i+1}=P_{i} \text {; } \\
& i=i+1 ;\}\}
\end{aligned}
$$

Fig. 1. Algorithm of ED-VTR that generates the transmission schedule for video traffic.

\section{Active video frame skip}

Involving in video streaming applications, insufficient decoder buffer capacity at client side may reduce the efficiency of traffic regulating and cause non-sustained video playback. Traditional methods such as the Work-Ahead Smoothing scheme (WAS) [4] alternatively increase and decrease the transmission rate by a wide margin for solving the problem. However, the operations may complicate the resource management of network, particularly when the available bandwidth is nearly exhausted. Instead of the above solution, ED-VTR includes an active video frame skip algorithm to solve the problem while still satisfying (3) and (4). The proposed frame skip algorithm can cooperate with ED-VTR to actively search for suitable frames to be skipped in advance at the sender, rather than passively and arbitrarily 
skipping overflowed frames in the event of network congestion or buffer overflow.

For the sake of explanation, both $L(\mathrm{n})$ and $U(\mathrm{n})$ are redrawn by simplified piece-wise linear segments, as shown in Fig. 2(a). By means of the proposed approach in Fig. 1, the raw transmission rate $\rho_{k}^{R}$ is obtained after executing the $k$-th linear concave upper approximation for the interval $\left[J_{k}, P_{k}\right]$ of $L(\mathrm{n})$. In Fig. 2(a), the concave depth $F_{l}$ is defined as the minimum distance between the point $L\left(\mathrm{~T}_{\mathrm{r}}\right)$ and the $k$-th linear concave upper bound. A large concave curvature is associated with a large concave depth. However, it is noted that a large concave depth combined with a small decoder buffer space increases the possibility of buffer overflow. Therefore, in the case involving in small decoder buffer space, the proposed frame skip algorithm is designed to reduce the concave depth and thus prevent possible decoder buffer overflow.

When the decoder buffer space is limited to $B$ bits, it is obvious that the buffer overflow occurs in the interval $\left[T_{0 l}, T_{o 2}\right]$ if $\rho_{k}^{R}$ is applied. Therefore, a new maximum permitted transmission rate without decoder buffer overflow for $\left[J_{k}, P_{k}\right]$ must be calculated by

$$
R_{\max }=\min _{J_{k}<\alpha \leq P_{k}}\left\{\frac{U(\alpha)-L\left(J_{k}\right)}{\alpha-J_{k}}\right\}
$$

The tangency point $T_{r}$ of $L(\mathrm{n})$ and the transmission schedule with $R_{\max }$ in $\left[J_{k}, P_{k}\right]$ can also be formulated as

$$
T_{r}=\left\{\tau \mid U(\tau)=R_{\max } \cdot\left(\tau-J_{k}\right)+L\left(J_{k}\right)\right\}
$$

On the other hand, although using $R_{\max }$ can prevent buffer overflow, it may also cause buffer underflow before time $P_{k}$. In Fig. 2(b), the first time instant that causes buffer underflow is denoted as $T_{u}$ and expressed as

$$
T_{u}=\min _{J_{k}<\tau \leq P_{k}}\left\{\tau \mid \frac{L(\tau)-L\left(J_{k}\right)}{\tau-J_{k}} \geq R_{\max }\right\}
$$

To identify the most suitable temporal range for skipping frames in $\left(J_{k}, T_{u}\right)$, this work first considers one of the possible temporal ranges, $\left(J_{k}, T_{r}\right)$. After skipping video frames in $\left(J_{k}, T_{r}\right)$, a new cumulative consumed function $L^{\mathrm{w}}(n)$ having the concave depth $F_{2}$ in $\left[J_{k}, P_{k}\right]$ is generated, as shown in Fig. 2(c). In $\left[J_{k}, T_{r}\right]$, the slope of $L^{\mathrm{w}}(n)$ is smaller than that of $L(n)$ because of frame skipping, but the slope of $L^{\mathrm{w}}(n)$ in $\left[T_{r}, P_{k}\right]$ is the same as that of $L(n)$. From Fig. 2(c), the concave depth $F_{2}$ is larger than $F_{l}$. That is, the operation of skipping frames in $\left(J_{k}\right.$, $T_{r}$ ] cannot achieve the target for reducing the concave depth but even worsen the problem. The above statement can be proved by simple triangle geometry. This work then considers another possible temporal ranges $\left[T_{r}, T_{u}\right]$ for effectively skipping frames. Using similar derivations, it easily can be proved that the concave depth in $\left[\mathrm{T}_{r}, \mathrm{P}_{k}\right]$ is effectively reduced if skipped video frames are located in interval $\left[T_{r}, T_{k}\right]$. Summarizing the above results, the most suitable temporal range for skipping frames in $\left(J_{k}\right.$, $P_{k}$ ) is limited to interval $\left[T_{r}, T_{u}\right]$.

Finally, from the viewpoint of the flexible temporal scalability in video coding characteristics, skipping B-frames can minimize the influence on received video quality. Therefore, B-frames are actively skipped in $\left[T_{r}\right.$, $T_{u}$ ] to remove the gap between $R_{\max }$ and $\rho_{k}^{R}$. The frame skip operation is repeated until the gap Diff between $\rho_{k}^{R}$ and $R_{\max }$ at time $T_{u}$ disappears, where Diff is displayed in Fig. 2(b). After accomplishing frame skip operations in $\left[T_{r}, T_{u}\right]$, the resulted traffic beginning from time $J_{k}$ is remodeled, and a mature traffic parameter pair $\left(\sigma_{k}^{T}, \rho_{k}^{T}\right)$ then is generated and added to $S^{T}(n)$.

\section{Simulation results and discussions}

In following simulation scenarios, this work assumes that ED-VTR is implemented in the video server where its output link bandwidth is $45 \mathrm{Mbps}$. Three compressed test video sequences, "Foreman", a part of movie "Jurassic Park I", and a part of movie "The Firm" are used. All video sequences are QCIF format with the frame rate of $30 \mathrm{fps}$. Moreover, a GOP consists of 15 frames and its pattern is set to I-B-B-P format. Besides, all sequences are

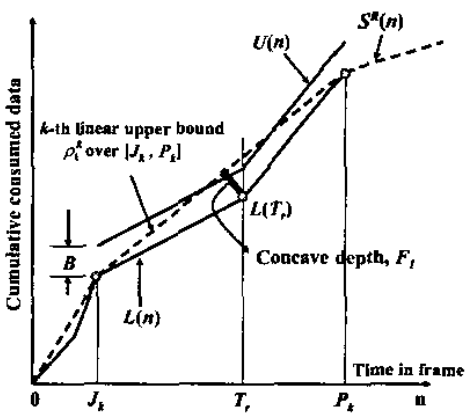

(a)

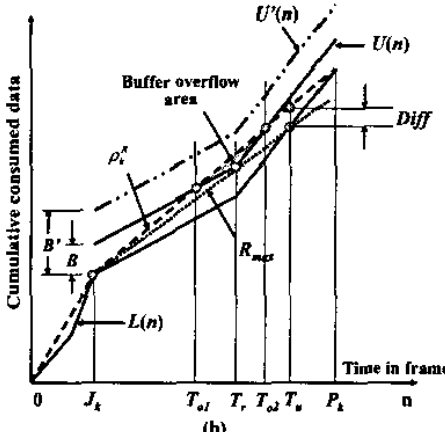

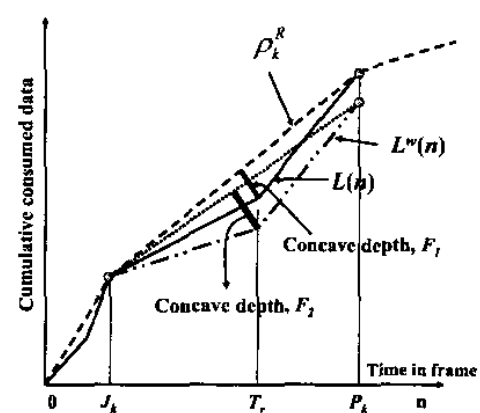

(c)

Fig. 2. Illustrations of active video frame skip algorithm: (a) simplified $L(n) \& U(n)$ with related parameters; (b) the region of buffer overflow and underflow; (c) the case of skipping frame in $\left(J_{k}, T_{r}\right]$. 
3 minutes in length, where "Foreman" is repeated cyclically to form the video sequence.

This work first utilizes three different video sequences to evaluate performances of ED-VTR. As shown in Fig. 3, ED-VTR can significantly reduce the peak bandwidth requirement in the initial stage, particularly for complex video sequences. The maximum outdoor rate $384 \mathrm{Kbps}$ of $3 \mathrm{G}$ networks is applied here as a referred upper bound of available network bandwidth. Moreover, from Fig. 4, the minimum required decoder buffer space for achieving loss free and bounded delay guarantees is presented. Based on three different video sequences, we observes that ED-VTR requires less decoder buffer space than the EEM method. To summarize, ED-VTR can effectively enhance the capability of EEM.

By observing curves in Fig. 5(a), both ED-VTR and WAS provide identical modeling performance given sufficiently large decoder buffer space. Nevertheless, if the decoder buffer space is insufficient with a short preloading time $D=2$, as in the case of Fig. 5(b), those bandwidth requirements generated from WAS oscillate during playback. In contrast, these bandwidth requirements generated from ED-VTR always keep the monotonically decreasing property. Note that the necessity of ED-VTR is gradually disappeared in the case of large $D$ that implies the availability of a large decoder buffer space. Briefly, ED-VTR can keep the advantage of WAS in cases of sufficiently large decoder buffer space, while enhancing the capability of WAS where decoder buffer space is insufficient.

\section{Conclusions}

The proposed ED-VTR mechanism can generate a series of monotonically decreasing bandwidth requests for a video bitstream, which can effectively reduce the complexity of resource management. Moreover, with insufficient decoder buffer space, ED-VTR can still maintain sustained playback with good picture quality by intelligent frame skipping. Simulation results show that ED-VTR can preserve the merits of traditional traffic modeling and smoothing methods yet increase the efficiency of bandwidth utilization.

\section{References}

[1] V. Frost and B.Melamed, "Traffic Modeling for Telecommunications Networks," IEEE Commun. Mag., vol. 32, no. 3, pp. 70-81, Mar. 1994.

[2] D. E. Wrege, E. W. Knightly, H. Zhang, and J. Liebeherr, "Deterministic Delay Bounds for VBR Video in Packet-Switching Networks: Fundamental Limits and Practical Trade-Offs," IEEE/ACM Trans. Networking, vol. 4, no. 3, Jun. 1996.

[3] I. M. Pao and M. T. Sun, "Encoding stored video for streaming applications," IEEE Trans. Circuit Syst. for Video Technol., vol. 11, pp. 199-209, Feb. 2001.
[4] J. D. Salehi, Z. L. Zhang, J. Kurose, D. Towsley, "Supporting Stored Video: Reducing Rate Variability and End-to-End Resource Requirements Through Optimal Smoothing," IEEE Trans. Networking, vol. 6, pp. 397-410, Aug. 1998.

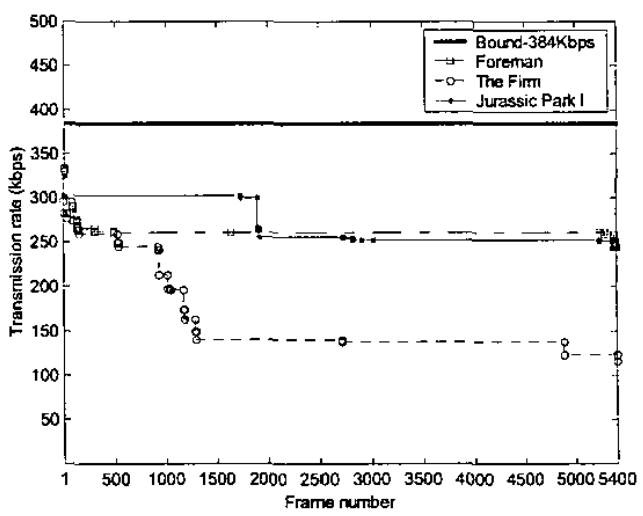

Fig. 3. Transmission rates generated by ED-VTR for various video sequences.

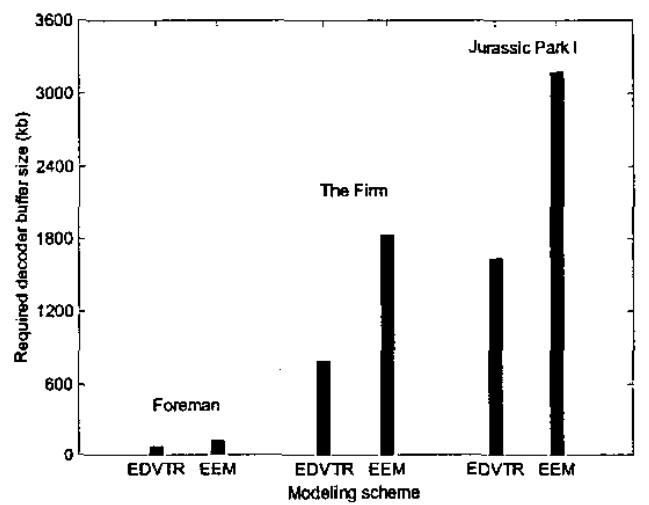

Fig. 4. Required decoder buffer space for achieving loss free and bounded delay guarantees.
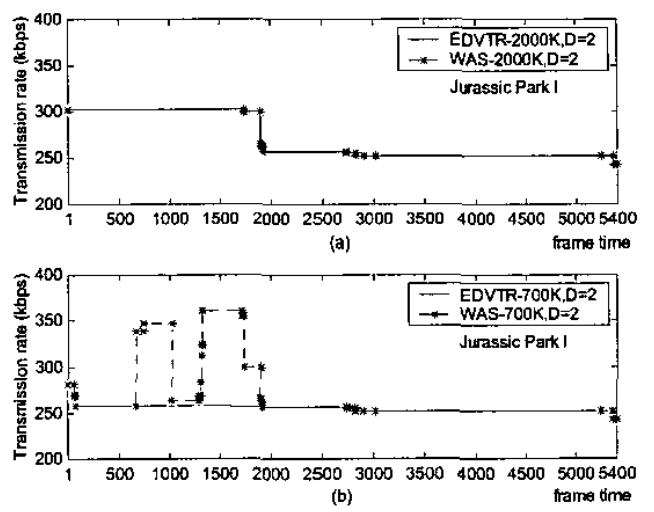

Fig. 5. Comparison between ED-VTR and WAS. (a) Buffer size $=2000 \mathrm{Kbits}, D=2$. (b) Buffer size = 700 Kbits, $D=2$. 